\title{
Hyperspectral Analysis of Cultural Heritage Artifacts: Pigment Material Diversity in the Gough Map of Britain
}

\author{
Di Bai ${ }^{\mathrm{a}}$, David W. Messinger ${ }^{\mathrm{b}}$, David Howell ${ }^{\mathrm{c}}$ \\ ${ }^{a}$ Chester F. Carlson Center for Imaging Science, Rochester Institute of Technology, Rochester, New York, 14623, \\ E-mail: db3641@rit.edu \\ ${ }^{\mathrm{b}}$ Chester F. Carlson Center for Imaging Science, Rochester Institute of Technology, Rochester, New York, 14623, \\ E-mail: messinger@cis.rit.edu \\ 'Bodleian Library, University of Oxford, Broad St, Oxford OX1 3BG, UK, E-mail: david.howell@bodleian.ox.ac.uk
}

\begin{abstract}
The Gough Map, one of the earliest surviving maps of Britain, was created and extensively revised over the 13th and 14th centuries. In 2015, map was imaged using a hyperspectral imaging system while in the collection at the Bodleian Library, Oxford University. The goal of the collection of the hyperspectral image (HSI) of the Gough Map was to address questions such as enhancement of faded text for reading and analysis of the pigments used during its creation and revision. In particular, pigment analysis of the Gough Map may help historians understand the material diversity of its composition and potentially the timeline of, and methods used in, the creation and revision of the map. Multiple analysis methods are presented to analyze a particular pigment in the Gough Map with an emphasis on understanding the within-material diversity, i.e., the number and spatial layout of distinct red pigments. One approach to understanding the number of distinct materials in a scene (i.e., endmember selection and dimensionality estimation) is the Gram Matrix approach. Here, this method is used to study the within-material differences of pigments in the map with common visual color. The application is a pigment analysis tool that extracts visually common pixels (here the red pigments) from the Gough Map and estimates the within material diversity of the pixels. Results show that the Gough Map is composed of at least 5 kinds of dominant red pigments with a particular spatial pattern. This research provides a useful tool for historical geographers and cartographic historians to analyze the material diversity of hyperspectral images of cultural heritage artifacts.
\end{abstract}

Keywords: hyperspectral imagery; pigment analysis; dimensionality estimation.

\section{Introduction}

The Gough Map, a mid-fourteenth century map of Britain, ${ }^{1-4}$ is the earliest surviving map of the island in geographically recognizable form. Previous research based on the visible features of the map revealed that this map was extensively revised after its original creation. ${ }^{1,3}$ The map contains many features, including over 600 town signs, a depiction of waterways, and distances between the towns. ${ }^{1-3}$ According to Delano-Smith, et al. (2017), the map was created in three distinct layers during three different periods, an initial creation step and two subsequent revision steps. The map appears to have been revised over approximately 100 years. However in each step similar pigments 
and methods were utilized to create and update the map, as evidenced by the homogeneity of signs and symbols on the map. ${ }^{3}$

Despite the interest in and study of the map, many questions still remain unanswered as to the method and motivation for its creation. In an effort to answer some of the questions related to the Gough Map, hyperspectral images of the map were collected at the Bodleian Library, Oxford University in 2015. The map was imaged in six "chips" to ensure sufficient spatial resolution while covering the entire map. Each image has 334 spectral bands over the Vis-NIR spectrum ranging from 400nm to $1000 \mathrm{~nm}$. The size of each chip is 1600 (pixels) x 5512 (pixels). More collection details will be stated in Section 3.1. It is hoped that analysis of the hyperspectral imagery will aid in the codicological studies of the map (i.e., understanding the tools, techniques, and timeline of how the map was created). This paper presents an approach to understanding one of the primary pigments used on the map, the red inks.

Like other cultural heritage studies, codicological questions arise from the Gough Map, such as the desire to read faded text and develop an understanding of the pigments used. Previous research in cultural heritage imaging has focused on the extraction of faded text using multispectral reflectance, fluoresence, and transmission imaging. ${ }^{5-13}$ Over several years, Easton and his collaborators have used a Megavision Imaging System to collect and generate multispectral images of artifacts and then used several image processing techniques to enhance the images. Methods used were principal component analysis, independent component analysis, unsupervised/supervised classification, spectral pseudoinverse calculation, deterministic renderings in pseudo-color, and spectral un-mixing to recover faded writings from historical manuscripts, such as the Archimedes Palimpsest and Herculaneum Papyri. ${ }^{5-13}$ For pigment analysis in cultural heritage, Comelli et al. (2007) developed a fluorescence multispectral imaging system to analyze surfaces of Renaissance 
frescoes. ${ }^{14}$ This system used UV lamps for excitation and a liquid crystal filter attached to a CCD as the detector. They acquired multispectral data by observing the fluorescence spectrum. Binders and pigments with specific fluorescence properties were detected and differentiated with this system. Zhao (2008) developed algorithms for image segmentation and pigment mapping for artistic paintings, such as "Starry Night", also based on multispectral imaging. ${ }^{15}$ Her methods included a spectral reconstruction algorithm based on Wyszecki's hypothesis and a pigment mapping algorithm based on Kubelka-Munk turbid media theory that can predict spectral reflectance for a mixed specimen from the optical properties of its constituent pigments. The output of the pigment mapping method was a sequence of spatial distributions of pigments composing the painting. Previous research on imaging of cultural heritage artifacts was based on multispectral imaging, but in this paper, we consider hyperspectral images (HSI) of the Gough Map. Specifically, we address the within material diversity of pigments in the HSI of the Gough Map to aid in understanding the construction methods of the map.

This paper is organized in the following way. Section 2 describes the approach used to estimate material diversity in hyperspectral images. Section 3 describes application of this technique to the hyperspectral imagery of the Gough Map and presents the results. Section 4 provides a summary of the results and findings.

\section{Material Diversity Estimation}

Dimensionality estimation can be used to understand the complexity and material diversity of hyperspectral data. An estimation of the dimensionality of the data set in the spectral domain is often assumed to be related to the number of distinct materials in the scene, a generally reasonable and applicable assumption. The dimensionality of a data set can be defined as the minimum num- 
ber of free variables required to represent the data without losing information. ${ }^{16}$ There are many approaches to estimating the dimensionality of a data set, but the most common in hyperspectral imagery is through Principal Components Analysis, equating the dimensionality of the data with the significance of a dimension in the transformed space through the magnitude of its associated eigenvalue. Other methods such as box-counting and point density have also been proposed. ${ }^{17}$

Here we use the approach developed by Messinger, et al. (2012) and applied by Canham, et al. (2011) to estimate the number of distinct materials in a hyperspectral scene. The approach utilizes the Gram Matrix, a similarity matrix representation of vector data that has unique properties useful when estimating high-dimensional data dimensionality. In this method, endmembers are extracted from the scene to represent the $k$ "pure" materials, and $k$ is chosen to be larger than an initial $a$ priori estimate of the number of materials (but not larger than the number of spectral bands in the image). We use the MaxD algorithm to automatically extract the $k$ endmembers from the scene. ${ }^{20}$ The Gram Matrix is an $m \times m$ matrix, where $m$ is the number of vectors in the set. The $(i, j)^{t h}$ element of the Gram matrix is given by the inner product of the $i$ and $j$ vectors in the set, such that

$$
G_{i, j}=\left\langle x_{i}, x_{j}\right\rangle
$$

The Gram Matrix has a useful proporty in that the determinant of $G$, termed the Gramian, is the square of the volume of the parallelepiped that encloses the vector set. Consequently, when the Gramian approaches zero, the vectors in the set are no longer linearly independent. For hyperspectral data this can be interpreted as the number of materials in the set that are unique if the Gram Matrix is constructed using the set of extracted endmembers. More details on how this approach can be used to estimate the number of unique materials in a hyperspectral scene can be found in 
the literature. ${ }^{18,19,21}$

In this paper, we first use a Mahalanobis distance classifier to classify each chip of the Gough Map into three parts, red pigment (towns and roads), green pigment (waterways) and gray background. In the analysis here, the pixels containing red pigments from the Gough Map are extracted and analyzed using the Gram Matrix approach described above. The goal is to estimate the number of red pigments utilized in the creation and revision of the Gough Map and to ascertain if there is a spatial pattern in the distribution of each pigment type across the map. This requires a within-class dimensionality estimation to separate out potentially different red pigments based on their spectra, despite their common visually "red" appearance.

\section{Application to the Gough Map}

As mentioned before, like other cultural heritage studies, questions arise from the Gough Map, such as enhancement of faded text and pigment analysis. In this paper, the objective is to estimate the within material diversity of the red pigments in the map through analysis of the hyperspectral imagery. Here we describe the application of the above-described analysis technique to the Gough Map, and in particular, the red pigments.

\subsection{Collection Parameters}

The actual size of the Gough Map is 55.3 x $116.4 \mathrm{~cm}$. As shown in Figs. 1 \& 2, it is drawn on two pieces of animal hide that were originally sewn together, but the stitching is barely visible. ${ }^{4}$ The scale is estimated at close to 1:1,000,000, or sixteen miles to one inch. 


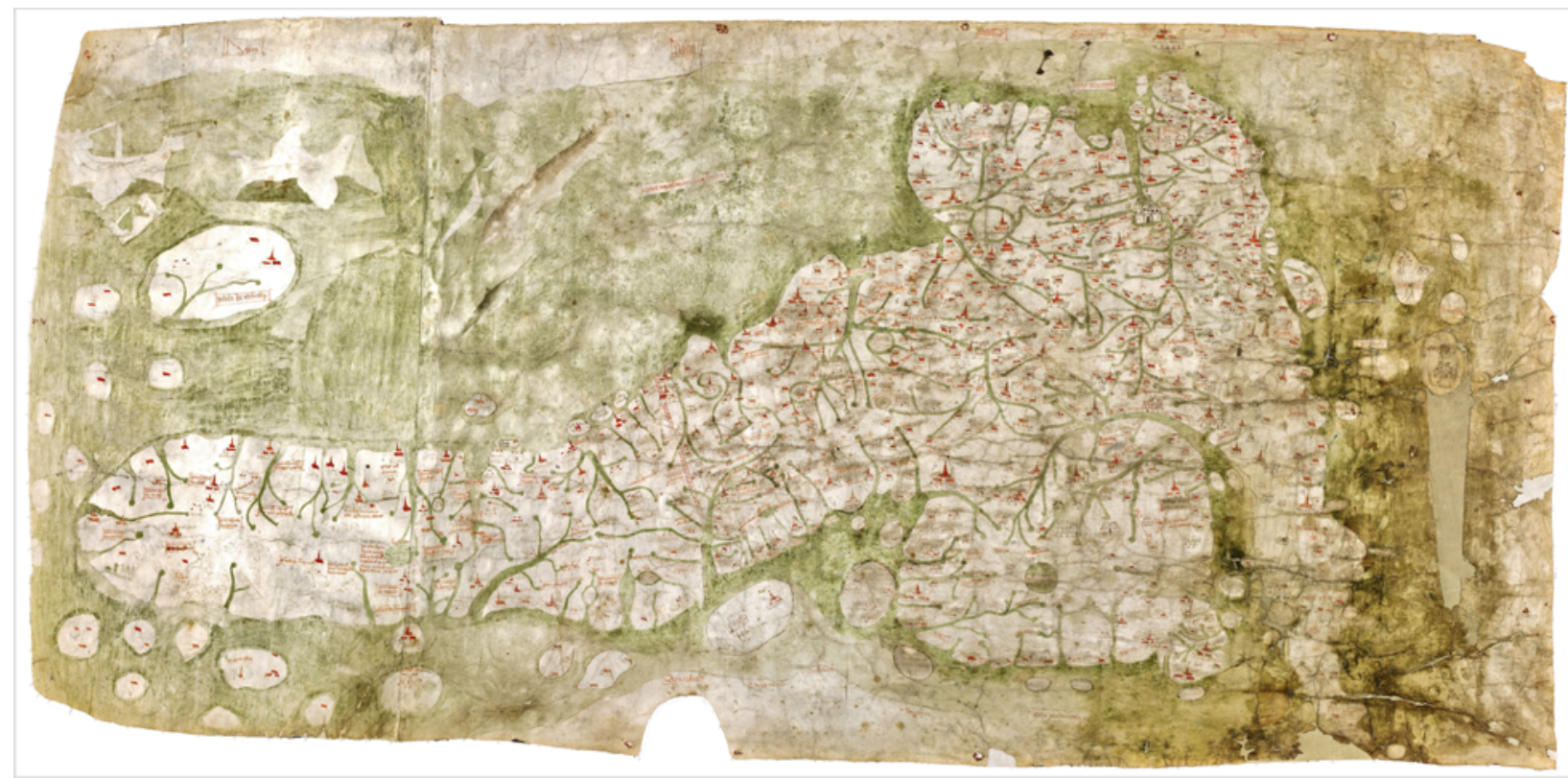

Fig 1 High resolution RGB image of the Gough Map. Note that the map is oriented such that East is at the top of the page and North (Scotland) is to the left. Towns are indicated by red signs; green represents water. The substrate of the map is lamb skin (leftmost quarter of map) and sheepskin (right 3/4 of map). Note the damaged area at the right edge (south of Wales).

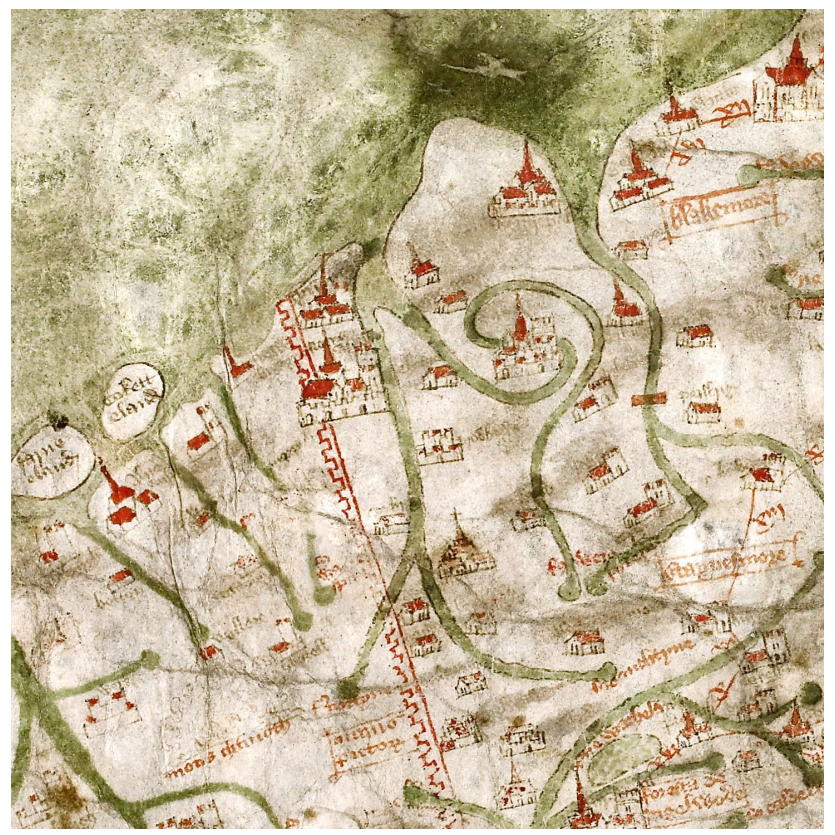

Fig 2 Zoom of the high resolution RGB image of the Gough Map showing town signs, distance indicators, labels, the coast line and waterways. Hadrian's Wall separating England and Scotland s also shown.

To ensure full coverage of the map with sufficient spatial resolution, the image collection was 
done in 6 spatially overlapping chips. Fig. 3 shows the six chips of the Gough Map rendered as RGB images. The overlapping areas are apparent as are the supporting structures around the edges to keep the map relatively flat. For convenience, we label the six parts as $1 \mathrm{~L}, 1 \mathrm{R}, 2 \mathrm{~L}, 2 \mathrm{R}, 3 \mathrm{~L}$ and

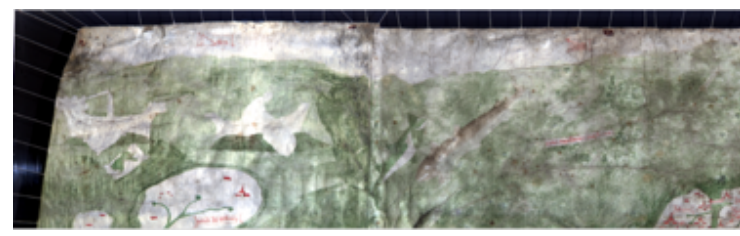

(a)

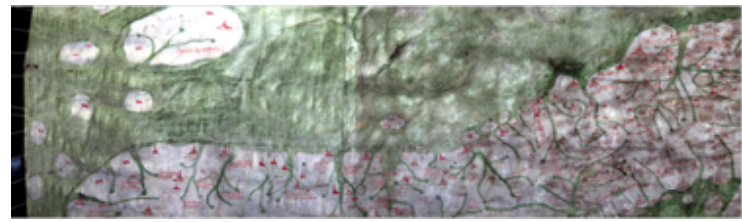

(c)

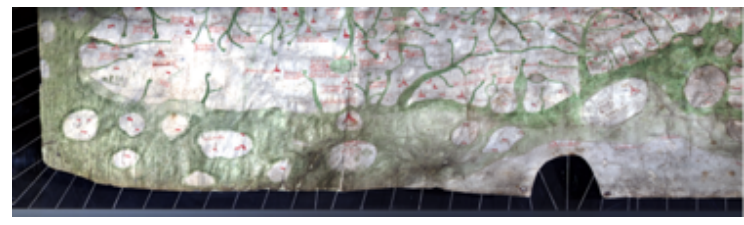

(e)

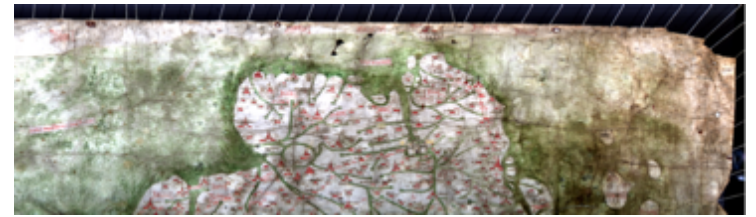

(b)

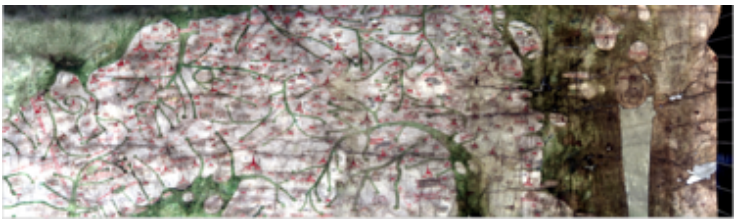

(d)

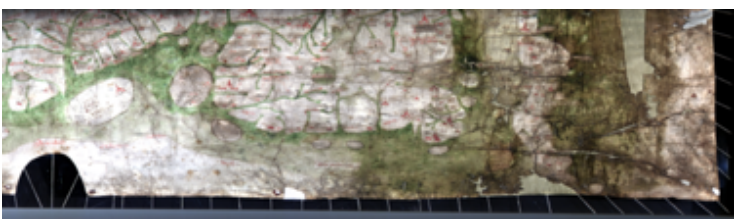

(f)

Fig 3 RGB renderings of the hyperspectral chips representing the collection of the entire Gough Map.

3R as shown in Fig. 4.

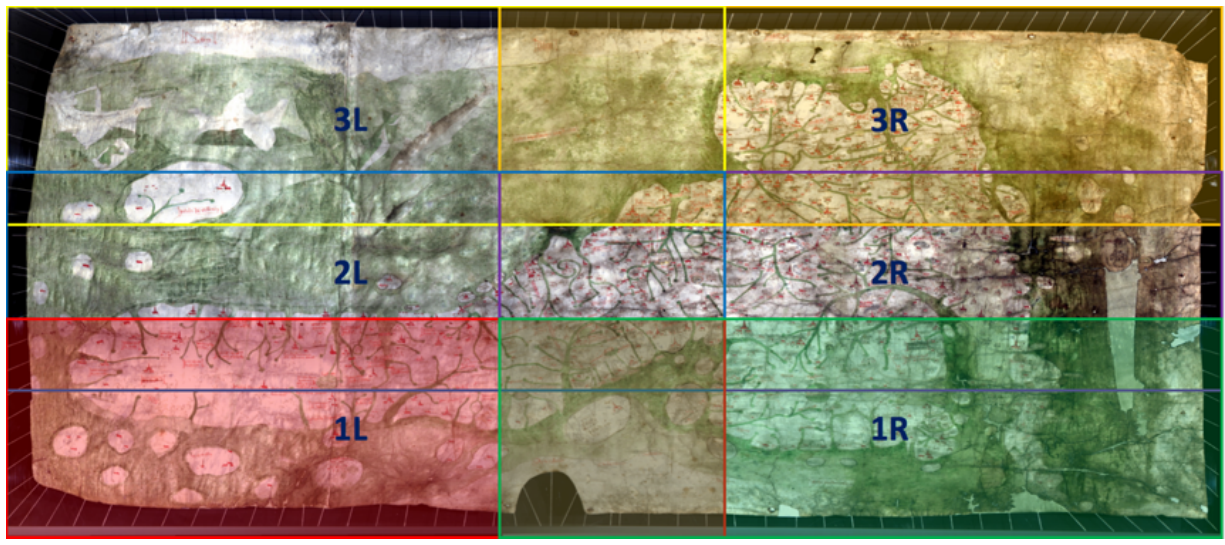

Fig 4 The entire Gough Map with chips labeled as 1L, 1R, 2L, 2R, 3L, 3R.

The size of each HSI chip is 1600 (pixels) x 5512 (pixels) x 334 (bands) and the whole Gough 
Map is about 3743 (pixels) x 8987 (pixels) x 334 (bands). Given that the actual size of the Gough Map is $55.3 \times 116.4 \mathrm{~cm}$, in the horizontal direction $\Delta x \simeq 77$ pixels $/ \mathrm{cm}$, and in the vertical direction $\Delta y \simeq 68$ pixels/cm. In addition, each HSI chip has 334 bands ranging from $398.7 \mathrm{~nm}$ to 1000.3 $\mathrm{nm}$ (VIS-NIR), so that $\Delta \lambda=1.8 \mathrm{~nm}$.

\subsection{Methodology}

We estimate the diversity of the red pigments in the Gough Map by using the techniques as described above. The methodology is outlined here and described in more detail below.

1. For each of the 6 chips in the hyperspectral image, sphere the data by unit magnitude normalizing each pixel to eliminate brightness differences in the estimated reflectance

2. Extract the red pixels of each chip using a Mahalanobis distance classifier on the (visually) red pixels

3. Use MaxD and the Gram Matrix to compute endmembers and estimate the dimensionality of the red pixels for each HSI chip

(a) extract $n$ endmembers $(n \approx 10)$

(b) iterate $j$ from 3 to $n$ endmembers

i. represent the $j$ endmembers in the Gram matrix

ii. compute the convex hull volume for $j$ endmembers by computing the Gramian

iii. save that volume as a function of $j$

(c) the estimated dimensionality is the number of endmembers where the volume function approaches zero 
4. For each chip, use spectral angle mapper to classify the red pixels using its endmembers as training data and count the number of pixels in each class

5. Estimate the global dimension of red pixels across all chips of the map

6. Map the results back to the image for visual interpretation

Details are provided below on the steps outlined above.

\subsubsection{Sphere data to eliminate brightness differences}

The first step is to sphere the data of each HSI chip of the Gough Map. In each collection, a white reference image was also collected and consequently, by normalizing out this white reference, the estimated reflectance of each pixel in the Gough Map was obtained. However, there appear to have been differences in this process and not all of the chips have the same brightness levels (shown below). Sphering the data, i.e., unit normalizing each pixel vector, is a way to remove brightness differences in the data set by ensuring each pixel has a vector magnitude of 1 . This produces a "normalized reflectance" for each pixel.

For example, Fig. 5 shows two images of a common area (the large island) from chips 3L (left) and $2 \mathrm{~L}$ (right). They come from the overlapping area between $2 \mathrm{~L}$ and $3 \mathrm{~L}$ on the Gough Map. Visually, $2 \mathrm{~L}$ looks brighter than $3 \mathrm{~L}$, and if we extract the same red pixel from both $2 \mathrm{~L}$ and $3 \mathrm{~L}$ and plot their spectra, the result shows that their spectra have significant brightness differences (Fig. 6) but little color difference. As a comparison, after we sphere the spectra (see Figure 6(b)), the brightness differences are removed. 


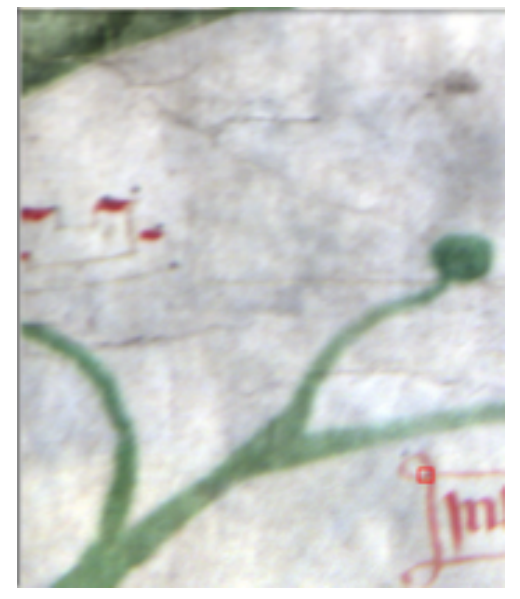

(a)

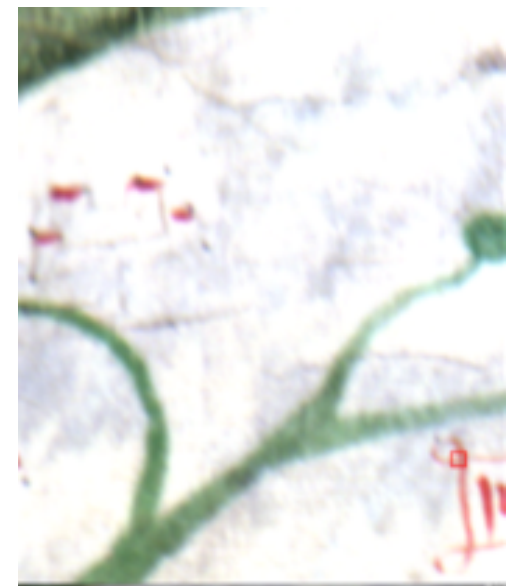

(b)

Fig 5 Example of same area in two different chips of the Gough Map: (a) $3 \mathrm{~L}$ and (b) $2 \mathrm{~L}$. Note the brightness differences when same histogram stretch is applied.

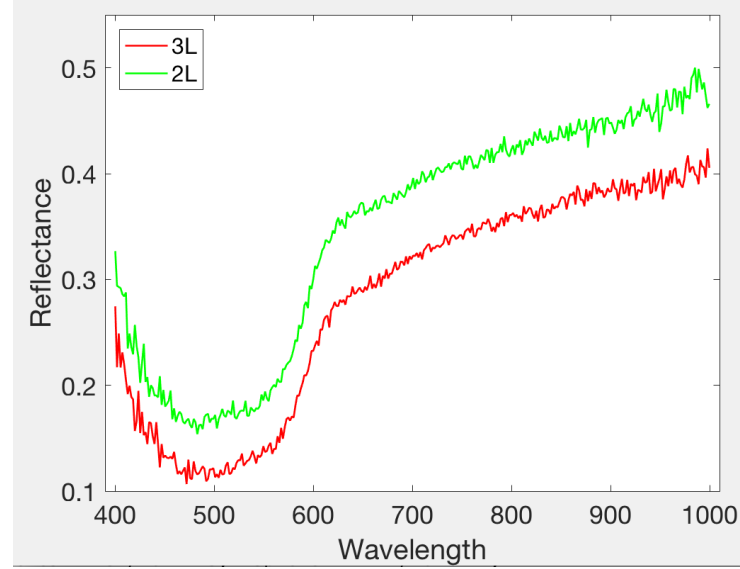

(a)

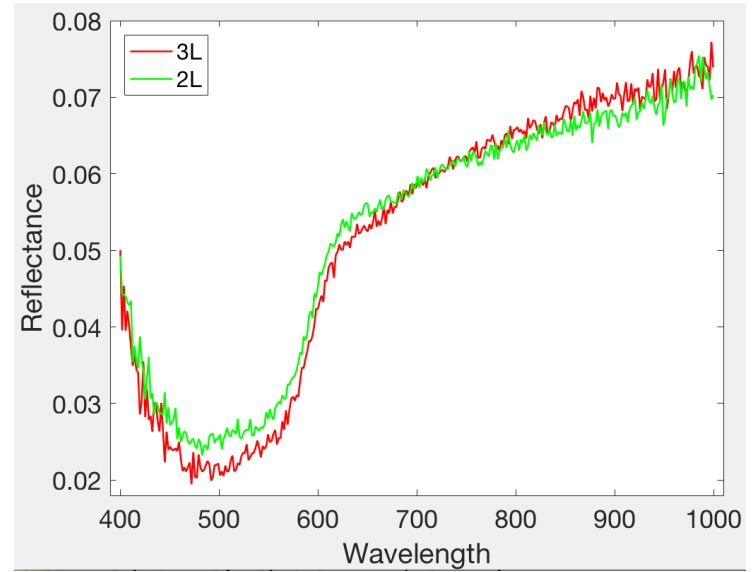

(b)

Fig 6 Spectral differences of common red pixel between $2 \mathrm{~L}$ and $3 \mathrm{~L}$ (a) before sphering and (b) after sphering.

\subsubsection{Extract red pixels}

The second step is to extract red pixels from the six HSI chips of the Gough Map. Since the 3L chip has very few red pixels, we discard it in the remaining analysis in this paper (see Figure 4). Here we use the ENVI software to classify the chips. For example, in the 2R chip as shown in Fig. 7(a), we first specify three regions of interest (ROI), the green pigment region, the red pigment region and the white background (the skin substrate), and then use a Mahalanobis distance classifier to 
classify this HSI chip. Fig.7(b) shows the result of the classification as a gray scale image, which is composed of three regions: black (green pigment), white (background), and gray (red pigment). Then we simply use a threshold to convert (b) to a binary image shown in Fig. 7(c), so that the white pixels in (c) represent all the red pixels in the original $2 \mathrm{R}$ chip, automatically extracted. The coordinates of all the identified red pigment pixels in Fig. 7(c) are saved (for future mapping) and the red pixel data are exported for within-material spectral analysis.

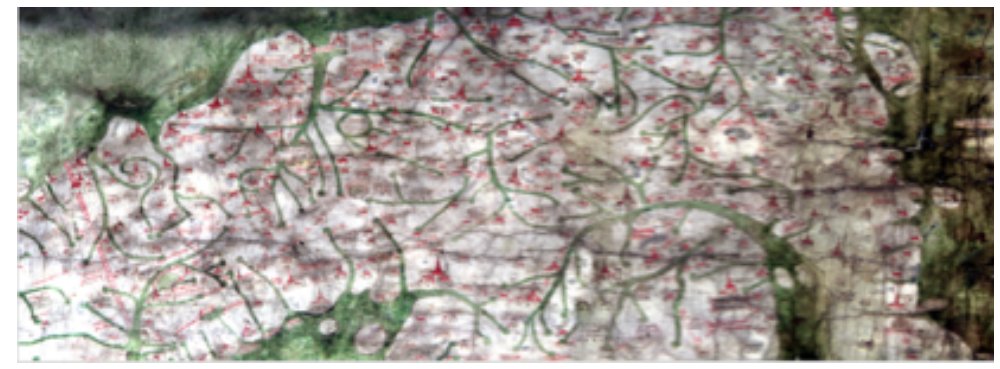

(a)

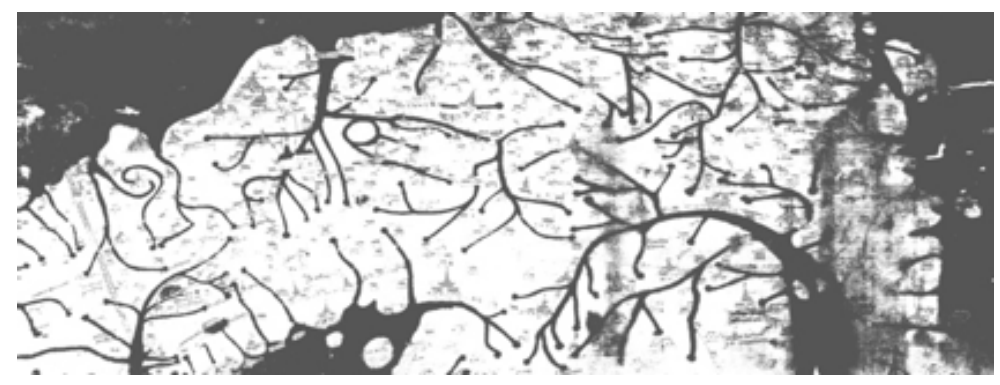

(b)

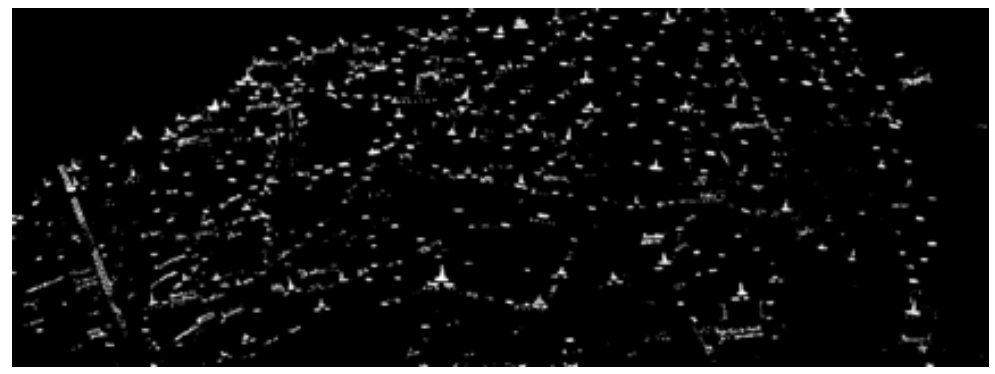

(c)

Fig 7 Example process of extracting red pixels in one chip of the Gough Map. (a) Original 2L chip from the Gough Map (b) Three class Mahalanobis classification result of the $2 \mathrm{~L}$ chip (c) binary image of $2 \mathrm{~L}$ with white pixels representing red pixels of the original $2 \mathrm{~L}$ chip. 


\subsubsection{Extract endmembers and estimate material diversity}

The third step is to use MaxD and the Gram Matrix approach to compute endmembers and estimate the number of distinct red pigments used for the red pixels of each HSI chip. In Fig. 8, and as described in Messinger et al., (2012), shown are the plots of the estimated convex hull volume as a function of increasing number of endmembers (dimensionality) of the data. Remember that the dimensionality at which the volume function approaches zero indicates the number of distinct materials in the scene. All of the five chips show that when 6 endmembers are used to represent the dat in the convex hull the estimated volume approaches zero, indicating 5 distinct red pigments in each chip. The similarity in these figures between chips is expected given the common materials and methods likely used across the entire map.

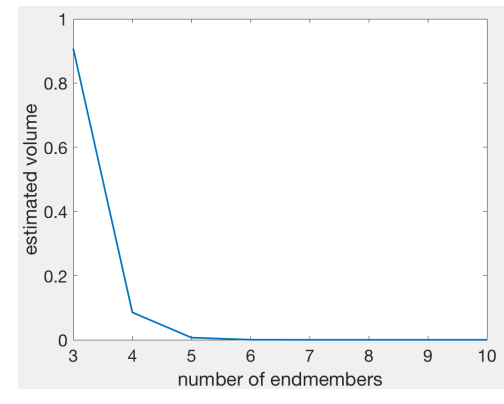

(a)

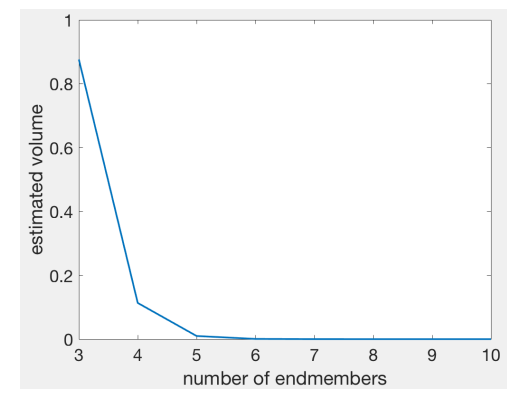

(b)

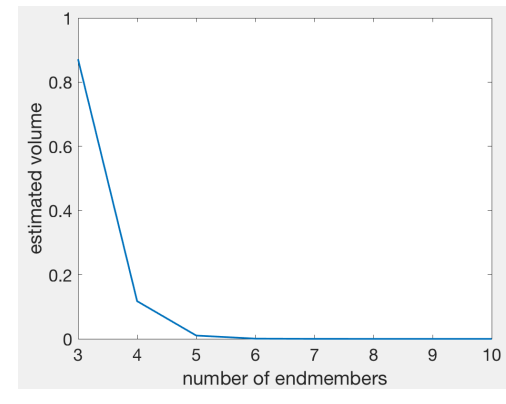

(c)

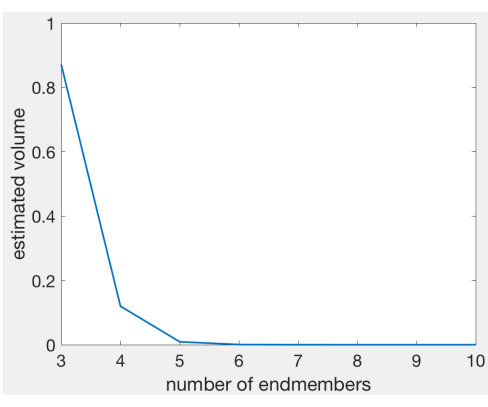

(d)

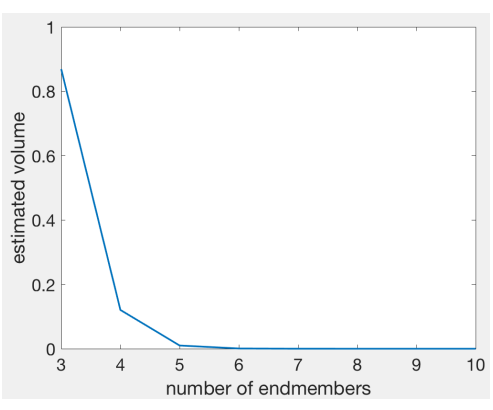

(e)

Fig 8 Complexity plots for the Gough Map: (a) 1L (b) 1R (c) 2L (d) 2R and (e) 3R with $x$ axis: number of endmembers and y axis: estimated convex hull volume.

Since the dimensionality of the red pixels in each HSI chip is 5, we output those five endmem- 
bers for each chip and plot them in Fig. 9(a-e). Those endmembers are actual red pixel spectra in each corresponding chip. We note that the five endmembers for each chip are distinct from each other and represent five unique material spectra per chip.

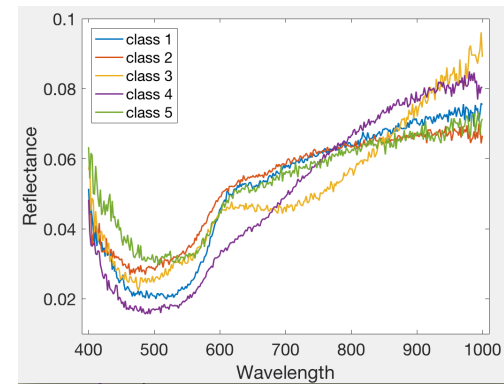

(a)

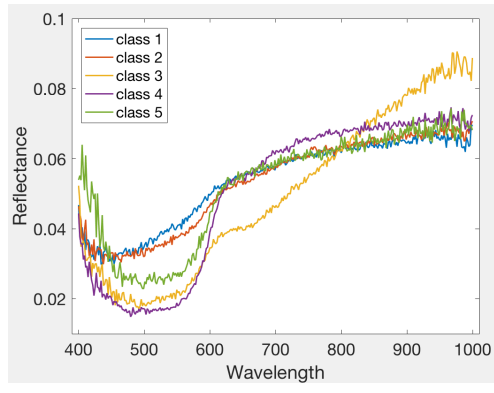

(b)

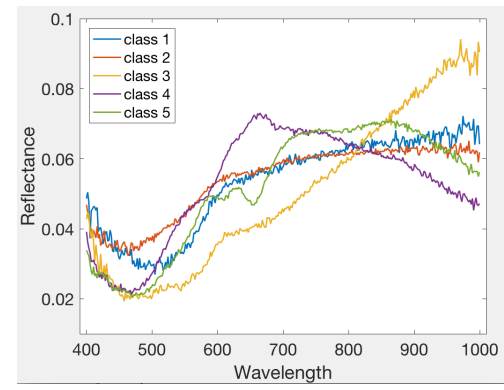

(c)

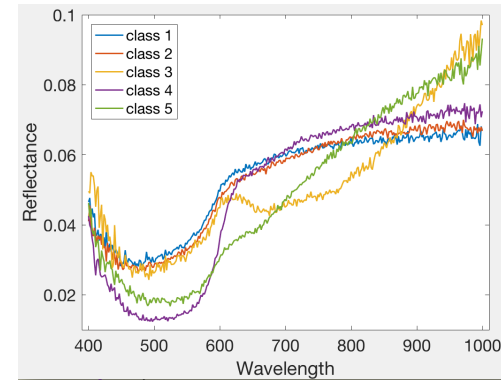

(d)

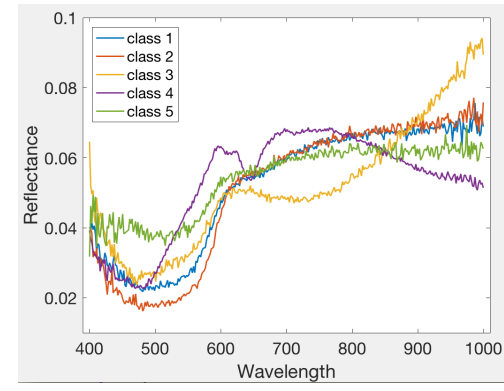

(e)

Fig 9 Endmember plots for the 5 chips of the hyperspectral image: (a) $1 \mathrm{~L}$ (b) $1 \mathrm{R}$ (c) $2 \mathrm{~L}$ (d) $2 \mathrm{R}$ and (e) $3 \mathrm{R}$ with $\mathrm{x}$ axis as wavelength in $\mathrm{nm}$ and $\mathrm{y}$ axis as reflectance.

\subsubsection{Classify the red pixels per chip}

The fourth step is to classify the red pixels based on the extracted endmembers per chip. We use the spectral angle mapper (SAM) to classify the red pixels within each chip and count the number of pixels in each class. Spectral angle ${ }^{22}$ is defined as

$$
\theta(x, y)=\cos ^{-1}\left(\frac{\sum_{i=1}^{n} x_{i} y_{i}}{\left(\sum_{i=1}^{n} x_{i}^{2}\right)^{\frac{1}{2}} *\left(\sum_{i=1}^{n} y_{i}^{2}\right)^{\frac{1}{2}}}\right)
$$


where $x$ is the spectral vector of a red pixel, $y$ is the spectral vector of an endmember, and $n$ is the number of spectral bands (here $n=334$ ).

For example, the 1L chip has 5 endmembers representing five distinct classes. We calculate the spectral angle between each pixel and the five endmembers in $1 \mathrm{~L}$, and see which endmember has the smallest spectral angle (i.e., highest spectral similarity) to this pixel and label it as such. In this way, we classify all the red pixels in each chip and count the number of pixels for each class. The results are shown in Table 1.

Table 1 The number of pixels per class for each HSI chip of the Gough Map. Note that class names here are not common across the entire map and refer to the within-class chip classes.

\begin{tabular}{|l|l|l|l|l|l|}
\hline Class & $1 \mathrm{~L}$ & $1 \mathrm{R}$ & $2 \mathrm{~L}$ & $2 \mathrm{R}$ & $3 \mathrm{R}$ \\
\hline 1 & 31479 & 6276 & 148939 & 47845 & 60304 \\
\hline 2 & 93728 & 36394 & 16491 & 137051 & 34101 \\
\hline 3 & 89 & 1314 & 7029 & 174 & 252 \\
\hline 4 & 333 & 15334 & 1 & 35869 & 9 \\
\hline 5 & 745 & 4138 & 8723 & 5755 & 19809 \\
\hline SUM & 126374 & 63456 & 181183 & 226694 & 114475 \\
\hline
\end{tabular}

From Table 1, notice that each chip has some dominant classes, for example, in $1 \mathrm{~L}$, the first class and second class contain most of the red pixels. Even though the result of the dimensinoality estimation tells us that there are 5 different materials in the $1 \mathrm{~L}$ chip, after we count the number of pixels, we see that there are two dominant red materials in the 1L chip. For visual interpretation, we map the results back to the image as described below.

\subsubsection{Visual interpretation: mapping the results back}

The fifth step is to map the per chip results back to the image for visual comparison. The classification results for each HSI chip are shown in Figure 10. Class 1 is interpreted in red, class 2: 
blue, class 3: green, class 4: yellow, class 5: cyan. From the figures, we notice that 1L is mainly composed of two types of red pixels, $1 \mathrm{R}$ is primarily composed of two pigments, $2 \mathrm{~L}$ is dominated by one red pigment, $2 \mathrm{R}$ is composed of 3-4 pigments, and 3R has 2-3 kinds of red. However, these colors only present the classification within each chip. Next we analyze the similarities in the red pigments between the chips and across the entire Gough Map.

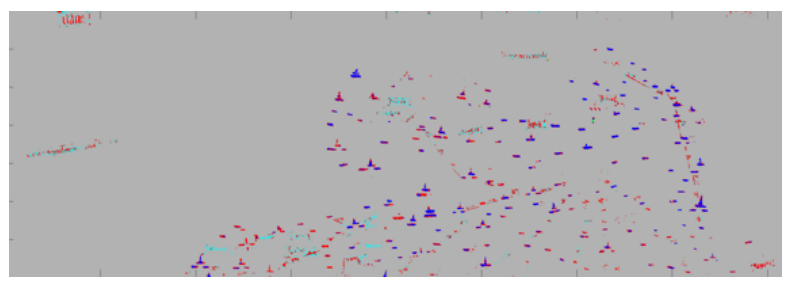

(a)

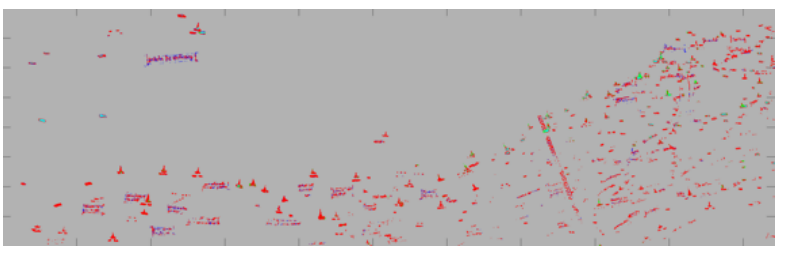

(b)

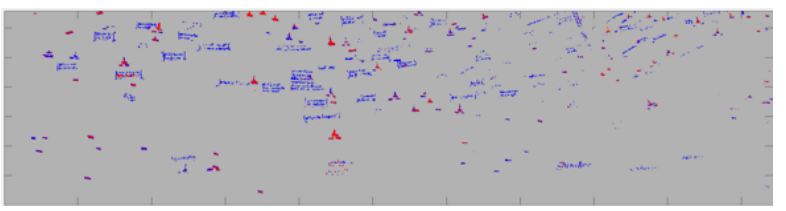

(d)

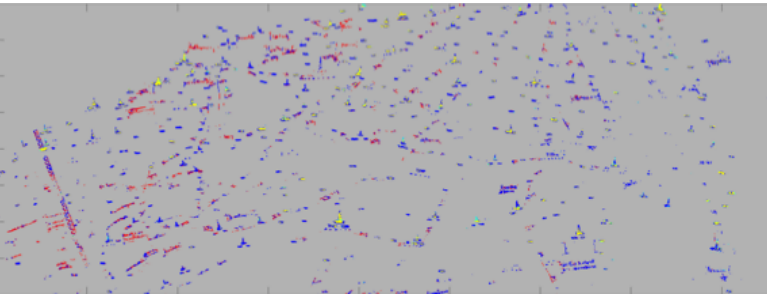

(c)

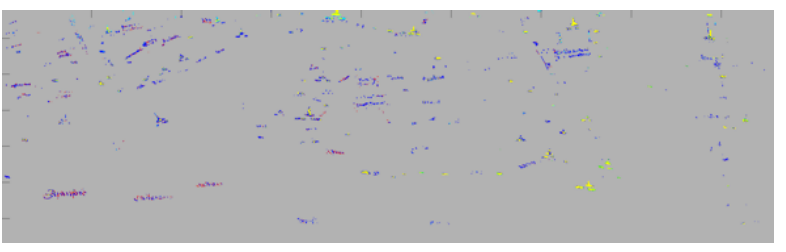

(e)

Fig 10 Classification results of each HSI chip of the Gough Map. (a) 3R (b) 2L (c) 2R (d) 1L and (e) 1R.

\subsubsection{Analyze spectral similarities between the HSI chips}

The last step is to estimate the number of red pigments in the entire Gough Map. To answer this question, we need to identify the dominant classes from each chip and consider their similarities. Here, we are only interested in the classes that have more than 10,000 pixels associated to their class in that chip. Consequently we only consider the following within-chip classes: 1L: class 1 , 
2; 1R: class 2, 4; 2L: class 1, 2; 2R: class 1, 2, 4; 3R: class 1, 2, 5. In total, we consider these twelve dominant classes from the individual chips. Now we need to combine similar classes. For example, in Fig. 10(b), class 1 in 2L is shown in red, while in Fig. 10(c), class 2 in 2R is shown in blue. From the two figures, we notice that there exist overlapping areas between $2 \mathrm{~L}$ and $2 \mathrm{R}$, where red in $2 \mathrm{~L}$ corresponds to blue in $2 \mathrm{R}$. A plot of these two endmembers is shown in Fig. 11. These two spectra are very similar to each other and so we assume are representative of the same material.

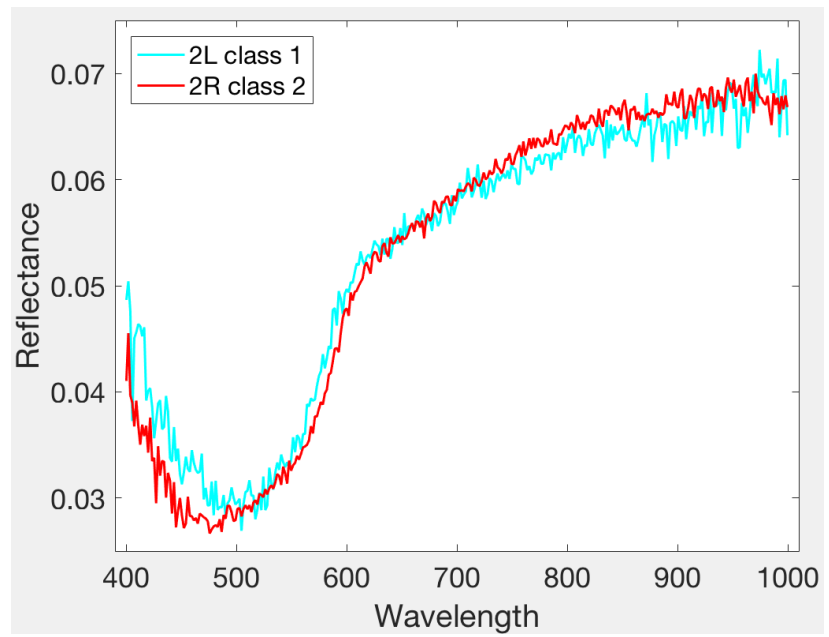

Fig 11 Example of spectra comparison between 2L class 1 in cyan and 2R class 2 in red.

Similarly, we found that $1 \mathrm{~L}$ : class $2,1 \mathrm{R}$ : class $2,2 \mathrm{~L}$ : class $1,2 \mathrm{R}$ : class $1-2$ and $3 \mathrm{R}$ : class 1 are also similar and will be clustered together as Pigment 1. Also 1L: class 1, 1R: class 4 and 3R: class 2 should be considered as a second unique cluster, called Pigment 2. Meanwhile, 2L: class 2, 2R: class 4 and 3R: class 5 are dissimilar from Pigments $1 \& 2$ and can be considered as separate materials, Pigments 3 - 5. Figure 12 shows the average spectra of the 5 pigments identified globally in the Gough Map. Notice that the spectra of the five dominant clusters are distinct from each other indicating that there are at least 5 distinct red pigment materials in the Gough Map.

Now we use the spectral angle to classify the red pixels over the entire map based on the five 


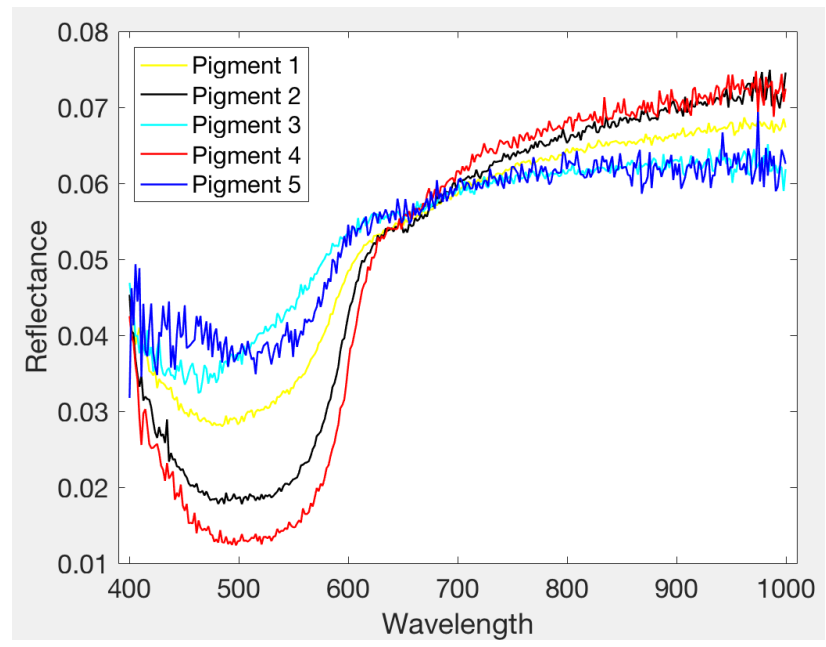

Fig 12 Spectra of five dominant classes of the red pixels in the Gough Map.

dominant classes and map the results back to the image for visual comparison. For convenience, we combine the five chips into one image of the entire Gough Map (remember that chip 3L had very few red pixels in it) and the classification result is shown in Figure 13. Class 1 is shown in red, class 2: blue, class 3: green, class 4: yellow, class 5: cyan. The number of pixels in class 1 is 423107, class 2: 228636, class 3: 49826, class 4: 4719, class 5: 5894, in total: 712182 pixels. From the figure, we notice that most of the towns are in blue, most of the texts are in red and some texts are in green. There are fewer pixels classified into final pigment classes $4 \& 5$ (yellow and cyan in the figure) due to the similarity of those classes to pigment 1 (see Figure 12). Consequently, while there are most likely five distinct red pigments, they are dominated by the two that make up most of the town signs and the writing. However, we also note that the majority of the pixels in pigment class $3 \& 4$ are found in the left portion of the map, suggesting an association with the Layer 1 construction based on the assessment of Delano-Smith, et al., (2017). Future analysis will consider these differences in either pigments used (e.g., was the pigment used for the writing "watered down" to flow through a quill pen) or tool usage (e.g., use of a pen for writing vs. a brush for the town signs). 


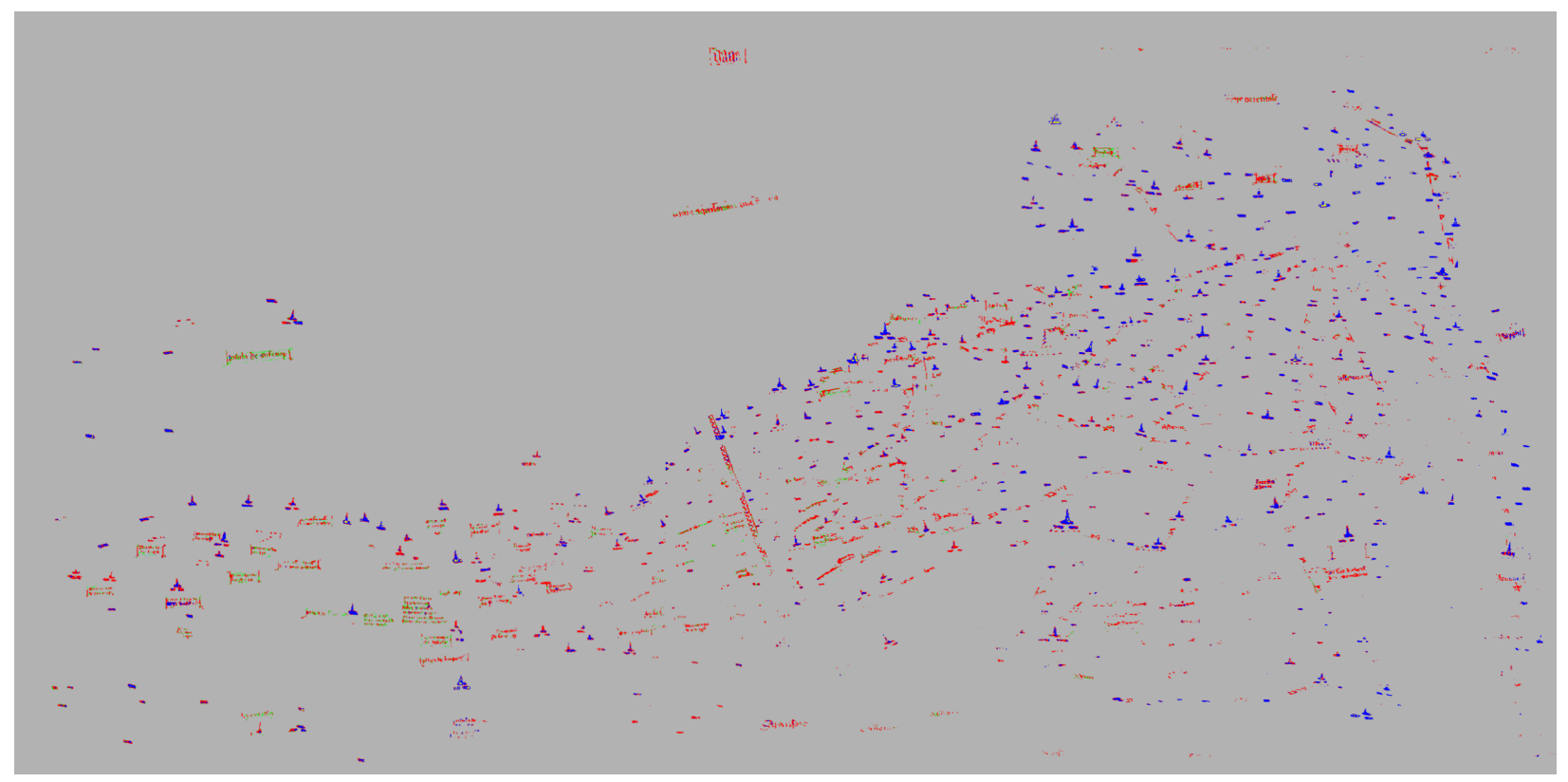

Fig 13 Classification result of HSI of the Gough Map (combination of five chips).

\section{Summary}

This work considers a novel application for hyperspectral imagery: analysis of pigment diversity in a medieval map. We have developed an approach to the analysis of pigments in a set of hyperspectral images of the Gough Map, the earliest surviving map of Britain in geographically recognizable form. The map was created and revised over an approximately 100 year period and the methods and motivation for its creation are largely unknown. This work analyzed the material diversity of the red pigments to aid in codicological studies of the map, i.e., understanding the tools, techniques, and materials used in its creation. Visually red pixels were extracted from each of the 6 image chips that compose the entire hyperspectral image. Endmember analysis and dimensionality estimation were performed per chip to understand the overall red pigment diversity across the map. These were then compared across the entire map and combined into global red pigment endmembers that are distinct materials. All red pixels in the map were then classified into one of these five 
global pigment classes and their spatial distribution was assessed by mapping them back into the large image so patterns are easily identified. Based on this analysis, we conclude that there are five unique red pigments present in the Gough Map. Additionally, we can confidently say that the primary differences in the red pigments are between the writing and the town signs and that there is some spatial relationship to the distribution of two of the pigment classes that could be related to the timeline of the construction and revision of the map. There could be many reasons for this including pigment and / or tool differences. This methodology, while here applied only to the red pigments to assess their within-material diversity, can be generalized to a novel pigment analysis tool for historical geographers and cartographic historians to analyze the material diversity of the hyperspectral images of other cultural heritage artifacts.

\section{Acknowledgments}

The authors wish to acknowledge Dr. Catherine Delano-Smith and Mr. Damien Bove for their insightful comments into the history, significance, and questions about the Gough map. We also wish to acknowledge Mr. Nick Millea at the Bodleian Library, Oxford University for his support and assistance with this project.

\section{References}

1 E. Solopova, "The making and re-making of the gough map of britain:manuscript evidence and historical context," Imago Mundi 64:2, 155-168 (2012).

2 K. D. Lilley, C. D. Lloyd, and B. M. S. Campbell, "Mapping the realm: A new look at the gough map of britain cartographic veracity in medieval mapping: Analyzing geographical variation in the gough map of great britain," Imago Mundi 61:1, 1-28 (2009). 
3 C. Delano-Smith, P. Barber, D. Bove, et al., "New light on the medieval gough map of britain," Imago Mundi 69:1, 1-36 (2016).

4 N. MILLEA, The Gough map: the earliest road map of Great Britain., The Bodleian Library, Oxford (2007).

5 R. L. Easton, W. A. C. Barry, and K. T. Knox, "Ten years of lessons from imaging of the archimedes palimpsest," Commentationes Humanarum Litterarum. 129, 5-34 (2011).

6 R. L. Easton and W. Noel, "Infinite possibilities: Ten years of study of the archimedes palimpsest," Proceedings of the American Philosophical Society 154, 50-76 (2010).

7 W. A. C. Barry, K. Boydston, and R. L. Easton, "Some properties of textual heritage materials of importance in spectral imaging projects," Commentationes Humanarum Litterarum. 129, 35-50 (2011).

8 W. A. C. Barry, K. Boydston, and R. L. Easton, "Some properties of textual heritage materials of importance in spectral 27 imaging projects," Proceedings of Eikonopoiia, Eikonopoiia, Digital Imaging of Ancient Textual Heritage: Technological Challenges and Solutions, Eikonopoiia 1, 27-38 (2010).

9 R. L. Easton, K. T. Knox, and W. A. C. Barry, "Some properties of textual heritage materials of importance in spectral 27 imaging projects," Proceedings of the European Signal and Image Processing Conference, Eurasip, 1440-1444 (2011).

10 K. T. Knox, R. L. Easton, W. A. C. Barry, et al., "Recovery of handwritten text from the diaries and papers of david livingstone," Proceedings of the SPIE, Electronic Imaging 7689 (2011). 
11 R. L. Easton and D. Kelbe, "Statistical processing of spectral imagery to recover writings from erased or damaged manuscripts," Manuscript Cultures. 7, 35-46 (2014).

12 R. L. Easton and W. Noel, "Multispectral imaging of the archimedes palimpsest," Gazette du Livre Medieval. 45, 39-49 (2004).

13 R. L. Easton, K. Sacca, G. Heyworth, et al., "Rediscovering text in the yale martellus map, spectral imaging and the new cartography," 7th IEEE International Workshop on Information Forensics and Security, 7th IEEE International Workshop on Information Forensics and Security (2015).

14 D. Comelli, G. Valentini, and R. Cubeddu, "Multi-spectral fluorescence imaging for cultural heritage," in Optics for Arts, Architecture, and Archaeology, C. Fotakis, L. Pezzati, and R. Salimbeni, Eds., Proc. SPIE 6618, 66180M1-66180M9 (2007). [doi:10.1117/12.726077].

$15 \mathrm{Y}$. Zhao, Image segmentation and pigment mapping of cultural heritage based on spectral imaging. PhD thesis, Rochester Institute of Technology, Rochester, NY (2008).

16 F. Camastra, "Data dimensionality estimation methods: A survey," Pattern Recognition 36, 2945-2954 (2003).

17 A. Schlamm, D. Messinger, and B. Basener, "Geometric estimation of the inherent dimensionality of single and multi-material clusters in hyperspectral imagery," Journal of Applied Remote Sensing 3 (2009).

18 D. W. Messinger, A. Ziemann, B. Basener, et al., "Metrics of spectral image complexity with application to large area search," Optical Engineering 51(3), 036201-1-036201-9 (2012).

19 K. Canham, A. Schlamm, A. Ziemann, et al., "Spatially adaptive hyperspectral endmem- 
ber selection and spectral unmixing," IEE Trans. on Geoscience and Remote Sensing 49(11) (2011).

20 J. R. Schott, K. Lee, R. Raqueno, et al., "A subpixel target detection technique based on the invariance approach," Proceedings of the AVIRIS Workshop, NASA, JPL, Pasadena, CA, (2003).

21 A. K. Ziemann, D. W. Messinger, and W. F. Basener, "Iterative convex hull volume estimation in hyperspectral imagery for change detection," Proc. SPIE 7695, 76951I-76951I-9 (2010).

22 F. A. Kruse, A. B. Lefkoff, J. B. Boardman, et al., "The spectral image processing system (sips) - interactive visualization and analysis of imaging spectrometer data," Remote Sensing of Environment. 44, 145-163 (1993). 